\title{
Ion and electron dynamics in the presence of mirror, electromagnetic ion cyclotron, and whistler waves
}

\author{
Jinsong Zhao, Tieyan Wang, Chen Shi, \\ Daniel B. Graham, Malcolm W. Dunlop, Jiansen He, \\ Bruce T. Tsurutani and Dejin Wu
}

\section{Published version information}

Citation: J Zhao et al. "Ion and electron dynamics in the presence of mirror, electromagnetic ion cyclotron, and whistler waves." Astrophysical Journal, vol. 883, no. 2 (2019): 185.

DOI: $10.3847 / 1538-4357 / a b 3 b d 1$

This version is made available in accordance with publisher policies. Please cite only the published version using the reference above. This is the citation assigned by the publisher at the time of issuing the APV. Please check the publisher's website for any updates. 


\title{
Ion and Electron Dynamics in the Presence of Mirror, Electromagnetic Ion Cyclotron, and Whistler Waves
}

\author{
Jinsong Zhao ${ }^{1}$ (1), Tieyan Wang ${ }^{2}$, Chen $\mathrm{Shi}^{1,3}$, Daniel B. Graham ${ }^{4}$, Malcolm W. Dunlop ${ }^{2,5}$, Jiansen $\mathrm{He}^{6}$, Bruce T. Tsurutani ${ }^{7}$, and \\ Dejin $\mathrm{Wu}^{1}$ \\ ${ }^{1}$ Key Laboratory of Planetary Sciences, Purple Mountain Observatory, Chinese Academy of Sciences, Nanjing 210008, People's Republic of China \\ js_zhao@pmo.ac.cn \\ ${ }^{2}$ RAL Space, STFC, Oxfordshire, OX11 0QX, UK \\ ${ }^{3}$ College of Astronautics, Nanjing University of Aeronautics and Astronautics, Nanjing, People's Republic of China \\ ${ }_{4}^{4}$ Swedish Institute of Space Physics, Uppsala, Sweden \\ ${ }_{6}^{5}$ School of Space and Environment, Beihang University, Beijing 100191, People's Republic of China \\ ${ }^{6}$ School of Earth and Space Sciences, Peking University, Beijing 100871, People's Republic of China \\ 7 Jet Propulsion Laboratory, California Institute of Technology, 4800 Oak Grove Drive, Pasadena, CA 91109, USA \\ Received 2019 July 1; revised 2019 August 13; accepted 2019 August 14; published 2019 October 3
}

\begin{abstract}
The wave-particle cyclotron interaction is a basic process in collisionless plasmas, which results in the redistribution of the energy between plasma waves and charged particles. This paper presents an event observation in order to explore the dynamics of charged particles and plasma waves, i.e., mirror, electromagnetic ion cyclotron (EMIC), and whistler waves, in the Earth's magnetosheath. It shows that when ions have a high-speed streaming velocity parallel to the magnetic field, EMIC waves arise. We also find that the frequency distribution of nearly parallel and nearly antiparallel whistler waves depends on the parallel streaming velocity of electrons. Based on the linear kinetic theory and the fitting plasma parameters, we show that the differential flows among ion components can enhance the ion cyclotron anisotropy instability that is even stronger than the mirror instability. The differential electron flows induce an asymmetry of the growth rate of counter-propagating whistler waves in the electron cyclotron anisotropy instability. On the other hand, the low-frequency EMIC and transverse electromagnetic waves modulate the ion pitch angle distribution. Moreover, when charged particles flow across the magnetic field, both low- and high-energy electrons are deeply trapped by mirror waves. These results illustrate new features of the observed plasma waves and charged particles in the Earth's magnetosheath, which could inspire improvement of the wave models therein.
\end{abstract}

Key words: instabilities - plasmas - waves

\section{Introduction}

The velocity distribution of charged particles in the solarterrestrial plasma environment can be determined by cyclotron interactions between particles and plasma waves. If plasma particles are out of the thermodynamic equilibrium, the instability of plasma waves could be triggered (e.g., Kennel \& Petschek 1966; Tsurutani \& Lakhina 1997), resulting in the system relaxing to a stable state. On the other hand, the energy of plasma waves can transfer to and from charged particles, broadening the velocity distribution and forming a plateau in the velocity distribution (e.g., Treumann \& Baumjohann 1997).

The planetary magnetosheath is full of various plasma waves and particles containing free energy, so it is a perfect laboratory to study the wave-particle interaction in collisionless plasmas. After high-speed particles in the solar wind cross the perpendicular portion of the planetary bow shock, both the plasma temperature and the temperature anisotropy of $T_{i \perp}>T_{i \|}$ are enhanced (e.g., Tsurutani \& Rodriguez 1981; Tsurutani et al. 1982; Price et al. 1986; Karimabadi et al. 2014; Hoilijoki et al. 2016). Another source of $T_{i \perp}>T_{i \|}$ comes from the magnetic field line draping in the magnetosheath (Midgley \& Davis 1963; Zwan \& Wolf 1976). The temperature anisotropy of $T_{i \perp}>T_{i \|}$ favors the generation of mirror mode (e.g., Hasegawa 1969; Tsurutani et al. 1982; Dimmock et al. 2015) and electromagnetic ion cyclotron (EMIC) waves (e.g., Kennel \& Petschek 1966; Gary 1992; Gary et al. 1993; Lee 2017; Zhao et al. 2019), where $T_{i \perp}$ and $T_{i \|}$ are ion temperatures perpendicular and parallel to the ambient magnetic field. Also, the electron temperature anisotropy of $T_{e \perp}>T_{e \|}$ in the magnetosheath can excite lion roars (e.g., Brice 1964; Smith et al. 1969; Smith \& Tsurutani 1976; Thorne \& Tsurutani 1981; Tsurutani et al. 1982; Lee et al. 1987; Zhang et al. 1998; Baumjohann et al. 1999; Maksimovic et al. 2001; Masood et al. 2006; Giagkiozis et al. 2018; Huang et al. 2018), which are the whistler wave mode in nature. The excitation of whistler waves can be closely related to the dynamics of the mirror mode (Tsurutani et al. 1982; Ahmadi et al. 2018; Breuillard et al. 2018). Electrons with pitch angles $\sim 90^{\circ}$ are heavily trapped in magnetic troughs associated with mirror mode waves, and these electrons are the source of whistler waves (Tsurutani et al. 1982; Ahmadi et al. 2018; Breuillard et al. 2018). Whistler waves can be also closely connected with the kinetic-scale magnetic holes (e.g., Huang et al. 2019), where the electron perpendicular anisotropy appears in magnetic dips relating to (electron-vortex) magnetic holes (e.g., Huang et al. 2017a, 2017b). Besides the temperature anisotropy, charged particles usually have different streaming velocities in the magnetosheath. These particle flows can considerably determine the properties of unstable plasma waves. For example, different growth rates for counter-propagating EMIC waves result from the ion differential flows (Zhao et al. 2019).

Using the Magnetospheric Multiscale Mission (MMS), this paper presents a clear observational evidence of the effect of the parallel ion/electron flow on the properties of basic plasma wave modes, i.e., mirror mode, EMIC, and whistler waves. Our event contains an obvious change of parallel ion and electron flows. Also, mirror mode, EMIC, and whistler waves coexist in 
this event. Interesting findings are that accompanying strong streaming ions and electrons, large-amplitude EMIC waves arise, and nearly parallel whistler waves have frequencies lower than nearly antiparallel whistler waves. Moreover, due to magnetic structures of mirror mode and EMIC waves, both ion and electron pitch angle distributions (IPDs and EPDs) are modulated in the observed event. Therefore, this study gives an excellent example for the wave-particle interaction in the magnetosheath.

In order to analyze the plasma dynamics and wave properties, we use magnetic field data from the FluxGate Magnetometer (Russell et al. 2016) and the Search Coil Magnetometer (Le Contel et al. 2016), electric field data from the Electric Double Probe (Ergun et al. 2016; Lindqvist et al. 2016), and particle data from the Fast Plasma Investigation (FPI; Pollock et al. 2016). This paper is organized as follows. Section 2 gives a comprehensive wave analysis. Section 3 shows the ion and electron dynamics in the presence of mirror mode, EMIC, and whistler waves. Section 4 presents instability analyses. The discussion and summary are included in Section 5.

\section{Wave Analyses}

Figure 1 presents an overview of our event, which occurs from 22:50:54.08 UT to 22:53:23.78 UT on 2016 January 18. This event was observed at $\sim[7.2,-9.1,-1.1] R_{\mathrm{E}}$ in geocentric solar ecliptic (GSE) coordinates, where $R_{\mathrm{E}}$ is the Earth radius. Magnetic field measurements in GSE coordinates show that $\left|B_{z}\right|>B_{y}>B_{x}>0$ in interval I (from 22:50:54.08 UT to 22:51:25.32 UT), $B_{x}>B_{y} \sim\left|B_{z}\right|$ in interval II (from 22:51:25.32 UT to 22:52:29.73 UT), and $\left|B_{z}\right|>B_{y} \sim$ $B_{x} \sim 0$ in interval III (from 22:52:29.73 UT to $22: 53: 23.78 \mathrm{UT}$ ). Three ion bulk velocity components always have the relation of $\left|V_{i x}\right|>\left|V_{i y}\right|>\left|V_{i z}\right|$ in the whole time interval. The angle $\theta_{B V_{i}}$ $\left(\theta_{B V_{e}}\right)$ between the magnetic field and the ion (electron) bulk flow is nearly $113^{\circ}$ in interval I, $134^{\circ}$ in interval II, and $90^{\circ}$ in interval III. It indicates that the parallel ion/electron flow in interval II is larger than that in interval I, and the ion/electron flow is nearly perpendicular to the magnetic field in interval III. Accompanying the large parallel ion/electron flows in interval II, there are clear signatures of nearly monochromatic fluctuations of the magnetic field and velocity. From Figure 1(d), the measured ion number density of $\sim 75 \mathrm{~cm}^{-3}$ is larger than the electron number density of $\sim 68 \mathrm{~cm}^{-3}$. This underestimation of the electron density may be caused by the failure to detect electrons with the energy lower than $\sim 10 \mathrm{eV}$. Figures 1 (e) and (f) show that both ion and electron particles have anisotropic temperatures - that is, the perpendicular temperature is larger than the parallel temperature. Using the definition of $\beta=2 \mu_{0} n\left(2 T_{\perp}+T_{\|}\right) /\left(3 B_{0}^{2}\right)$, the ion and electron plasma betas are $\beta_{i} \sim 3.6$ and $\beta_{e} \sim 0.7$, shown by Figure 1(g). Moreover, the typical energy for ions and electrons (Figures 1(h) and (i)) are about $200-1000 \mathrm{eV}$ and $50-100 \mathrm{eV}$, respectively. By comparing the magnetic field (Figure 1(a)) with plasma measurements (Figure 1(d)), the plasma density always enhances in magnetic field troughs to preserve total pressure balance, consistent with the feature of the mirror mode, which is analyzed in detail in Figure 2.

Figure 2 gives a comprehensive wave analysis. Since the timescale of magnetic fluctuations is normally smaller than $10 \mathrm{~s}$ (Figure 1(a)), the magnetic field data are smoothly averaged over $10 \mathrm{~s}$. This averaged magnetic field is used as the background magnetic field, and then the magnetic field and particle velocity in GSE coordinates are transformed into the field-aligned coordinate

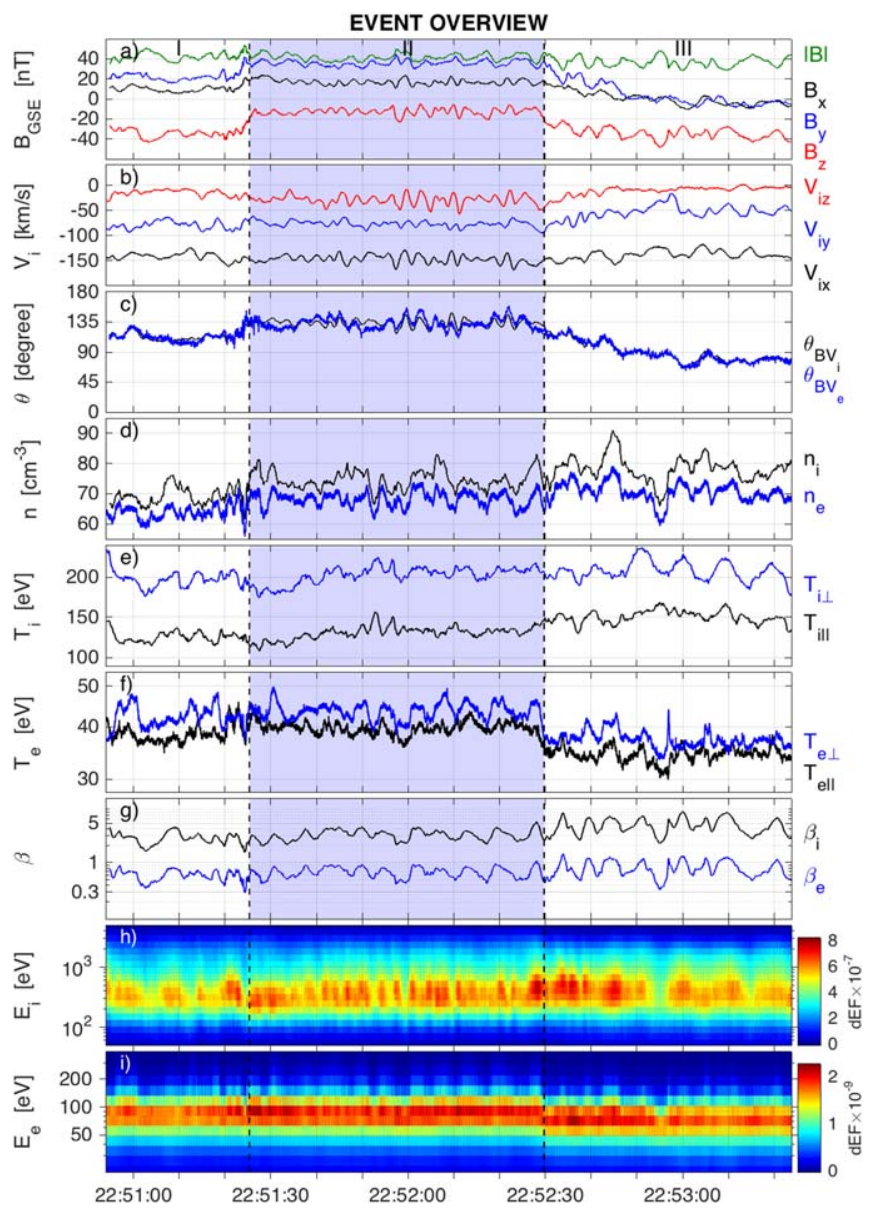

Figure 1. Event overview from MMS4 observations. (a) Magnetic field components in the GSE coordinates and the total magnetic field. (b) Ion bulk velocity components in GSE coordinates. (c) the angle $\theta_{B V_{i}}\left(\theta_{B V_{e}}\right)$ between the magnetic field and the ion (electron) bulk velocity. (d) Ion and electron number density. (e) Ion parallel and perpendicular temperature. (f) Electron parallel and perpendicular temperature. (g) Ion and electron plasma beta. (h) Ion differential energy flux. (i) Electron differential energy flux. The event time is divided as Interval I from 22:50:54.08 UT to 22:51:25.32 UT, Interval II from 22:51:25.32 UT to $22: 52: 29.73 \mathrm{UT}$, and Interval III from 22:52:29.73 UT to 22:53:23.78 UT

(FAC) systems. Figure 2(a) shows three magnetic field components in FAC. From the parallel magnetic field component $B_{\|}$, it clearly illustrates the existence of compressible magnetic field fluctuations. Also, transverse magnetic field fluctuations arise, and their amplitudes can reach $\sim 8 \mathrm{nT}$. To further identify the nature of transverse magnetic fluctuations, we compare normalized magnetic field $\boldsymbol{B} / B_{0}$ to normalized ion velocity $\boldsymbol{V}_{i} / V_{\mathrm{A}}$ (Figures 2(b)-(d)), where $\boldsymbol{B}$ and $\boldsymbol{V}_{\boldsymbol{i}}$ are the magnetic field and ion velocity filtered in the frequency range of $0.1-1 \mathrm{~Hz}$, and $V_{\mathrm{A}}=B_{0} / \sqrt{\mu_{0} n_{i} m_{p}}$ is the Alfvén speed. From Figures 2(b) and (c), we find $\boldsymbol{B}_{\perp} / B_{0} \simeq$ $\boldsymbol{V}_{i \perp} / V_{\mathrm{A}}$ in interval II, which is in accord with the theoretic prediction of the Alfvén/ion cyclotron wave propagating antiparallel to the magnetic field (Zhao 2015). In interval III, $\left|\boldsymbol{B}_{\perp}\right| / B_{0} \simeq\left|\boldsymbol{V}_{i \perp}\right| / V_{\mathrm{A}}$, and the relation between $\boldsymbol{B}_{\perp} / B_{0}$ and $\boldsymbol{V}_{\boldsymbol{i} \perp} / V_{\mathrm{A}}$ is mixed with positive-correlation, anticorrelation and $\pi / 2$ phase difference. However, there is no correlation between $\boldsymbol{B}_{\perp}$ and $\boldsymbol{V}_{\boldsymbol{i}}$ in interval I. From the analysis of the cross-correlation between $B_{\perp 1} / B_{0}$ and $V_{\mathrm{i} \perp 1} / V_{\mathrm{A}} \quad\left(B_{\perp 2} / B_{0}\right.$ and $\left.V_{\mathrm{i} \perp 2} / V_{\mathrm{A}}\right)$, the correlation coefficient $\mathrm{CC} \simeq 0.69$ (0.65) in interval $\mathrm{II}$ is larger than $\mathrm{CC} \simeq$ $0.24(0.30)$ in interval $\mathrm{I}$ and $\mathrm{CC} \simeq 0.17(0.13)$ in interval III. 


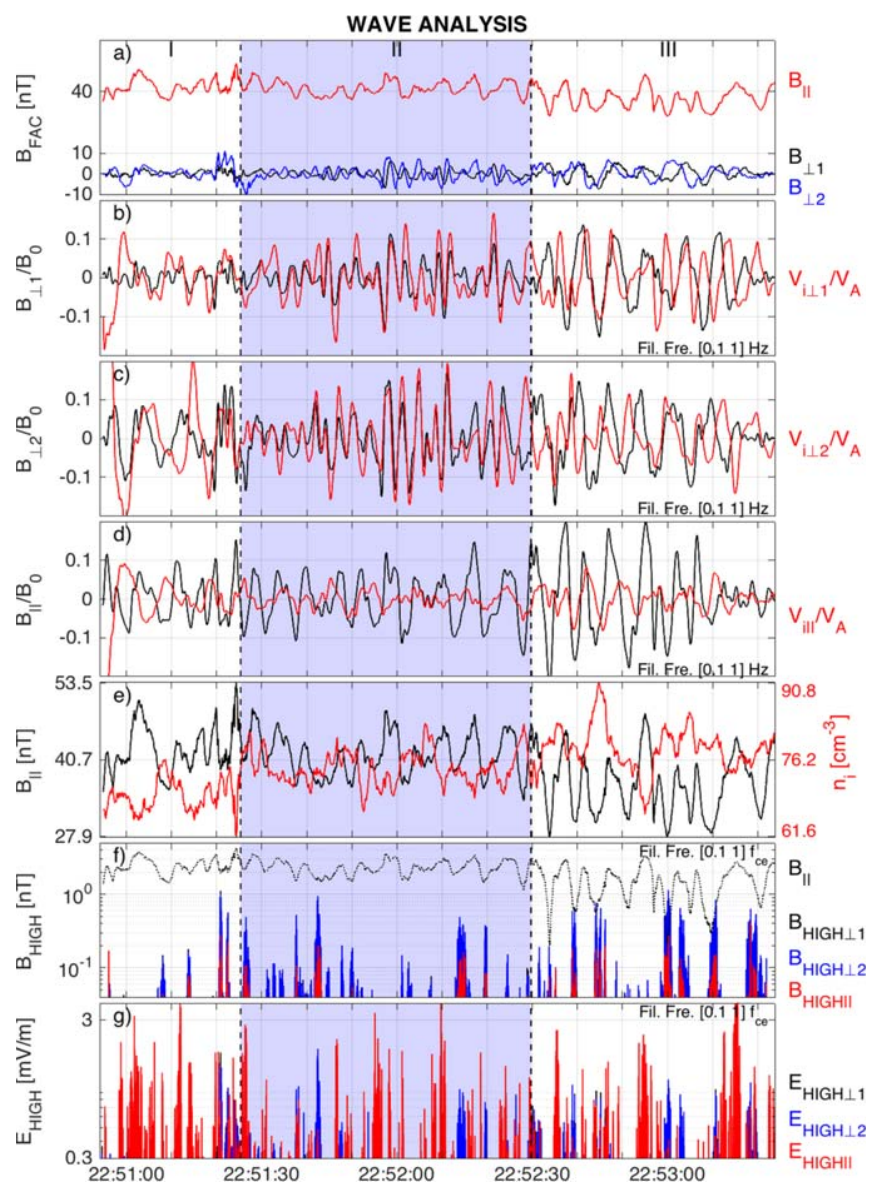

Figure 2. Wave analyses from MMS4. (a) Magnetic field components in the FAC. (b) and (c) Perpendicular magnetic field components (normalized by $B_{0}$ ) and perpendicular ion velocity components (normalized by $V_{A}$ ), where the filter frequency range is from 0.1 to $1 \mathrm{~Hz}$. (d) Parallel magnetic field component (normalized by $B_{0}$ ) and parallel ion velocity (normalized by $V_{A}$ ), where the filter frequency range is from 0.1 to $1 \mathrm{~Hz}$. (e) Parallel magnetic field and ion number density. (f) High-frequency magnetic field components in FAC, where the filter frequency range is $\left[\begin{array}{ll}0.1 & 1\end{array}\right] f_{\text {ce. }}$ (g) High-frequency electric field components in FAC, where the filter frequency range is $\left[\begin{array}{ll}0.1 & 1\end{array}\right] f_{\mathrm{ce}}$.

For $\boldsymbol{B}_{\|}$and $\boldsymbol{V}_{i \|}$, they have no clear correlation $(\mathrm{CC} \simeq-0.13)$ in the whole time interval. Figure 2(e) compares the parallel magnetic field $B_{\|}$to the ion density $n_{i}$. $B_{\|}$is negatively correlated with $n_{i}$, which results from the mirror mode. Furthermore, low-frequency compressible magnetic field fluctuations have a close relation with high-frequency magnetic and electric field fluctuations, as shown in Figures 2(f) and (g) where the magnetic and electric fields are filtered in the frequency range of $0.1 f_{\mathrm{ce}}-f_{\mathrm{ce}}$. Normally, in magnetic troughs, transverse magnetic and electric field fluctuations are stronger than parallel counterparts, $B_{\mathrm{HIGH} \perp} \gg B_{\mathrm{HIGH} \|}$ and $E_{\mathrm{HIGH} \perp} \gg E_{\mathrm{HIGH} \|}$. Besides, there are quasi-electrostatic fluctuations in our event, where $E_{\mathrm{HIGH} \|} \gg E_{\mathrm{HIGH} \perp}$ and $B_{\mathrm{HIGH}}$ is weak; however, they have no relation with the low-frequency compressible magnetic fluctuations.

Figure 3 further gives polarization analyses of highfrequency electromagnetic fluctuations using the singular value decomposition method (Santolík et al. 2003). Figures 3(a) and (b) present power spectral densities of the total magnetic and electric fields. There are strong electromagnetic field emissions in the frequency range of $0.1 f_{\text {ce }}-f_{\text {ce }}$, which are obviously distinct from the strong electrostatic field emissions that have

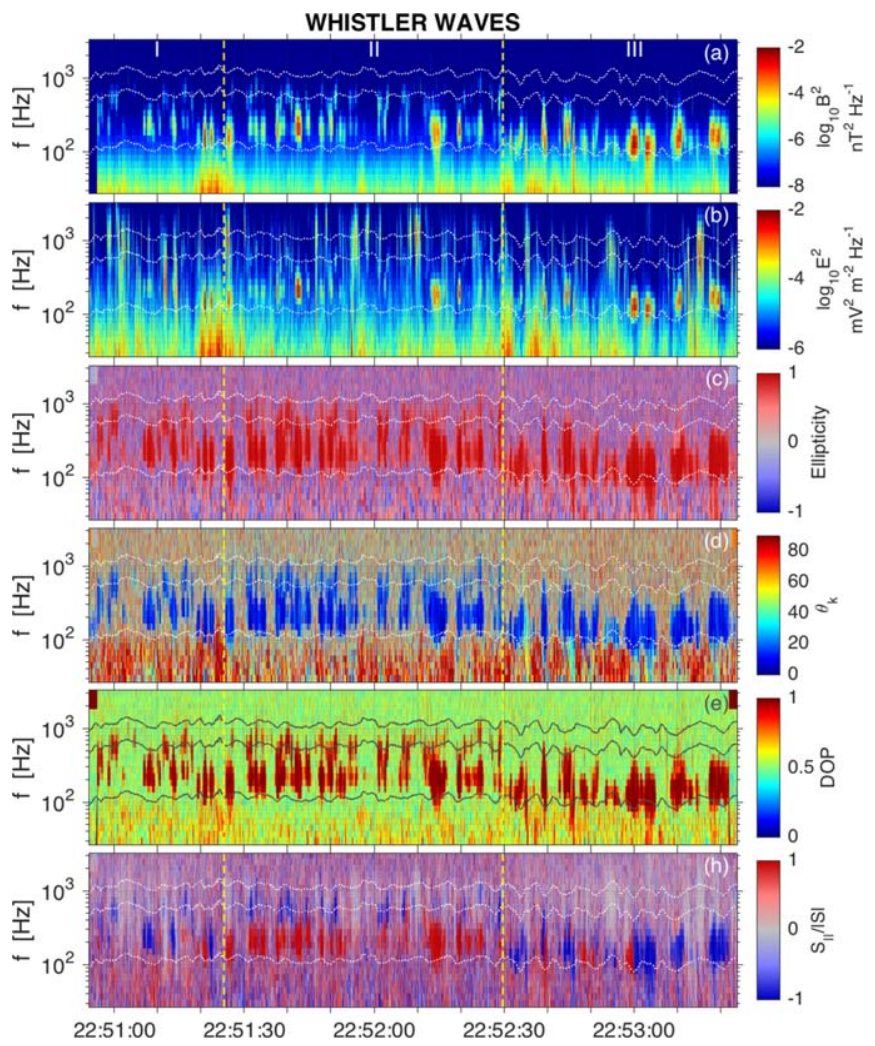

Figure 3. Polarization analyses in the frequency range of $\left[\begin{array}{ll}30 & 3000] \\ \mathrm{Hz}\end{array}\right.$ (a) Magnetic field power spectral density. (b) Electric field power spectral density. (c) Ellipticity. (d) Angle between the background magnetic field and wave vector. (e) DOP. (h) Poynting flux along the background magnetic field. Three lines overlaid in figures denote three characteristic frequencies of $0.1 f_{\text {ce }}$, $0.5 f_{\text {ce }}$, and $f_{\text {ce }}$.

the frequency extending from $f \ll 0.1 f_{\text {ce }}$ to $f \gg f_{\text {ce }}$. These strong electromagnetic emissions have high degree of polarization (DOP, \0.8) and high ellipticity ( $\gtrsim 0.8)$, indicating approximately right-hand circular polarization. They are nearly parallel $\left(\theta_{k} \lesssim 20^{\circ}\right.$ and $\left.S_{\|} /|S|=1\right)$ and antiparallel propagation $\left(160^{\circ} \lesssim \theta \lesssim 180^{\circ}\right.$ and $\left.S_{\|} / S \mid=-1\right)$. Therefore, these highfrequency electromagnetic fluctuations are whistler waves. The most interesting finding is that the wave frequency has different distributions in different time intervals. In interval II, the frequency of nearly parallel whistler waves are lower than that for nearly antiparallel waves. In interval III, counter-propagating whistler waves are mixed in the same frequency band. The above two frequency relations between counter-propagating whistler waves arise in interval I. Comparing Figures 3(h) to 1(c), we propose that the frequency distribution of whistler waves is strongly dependent upon the parallel electron streaming velocity.

\section{Particle Dynamics}

\subsection{Ion Dynamics}

The IPD is presented in Figure 4. It clearly shows different pitch angle distributions in different time intervals. In interval I, ions usually enhance in the pitch angle range of $\sim 90^{\circ}-135^{\circ}$, whereas in several short time intervals (i.e., 22:51:00 UT, 22:51:08 UT, 22:51:12 UT, and 22:51:18 UT), a part of the ions can extend to $180^{\circ}$. In interval II, the modulation of the ion 


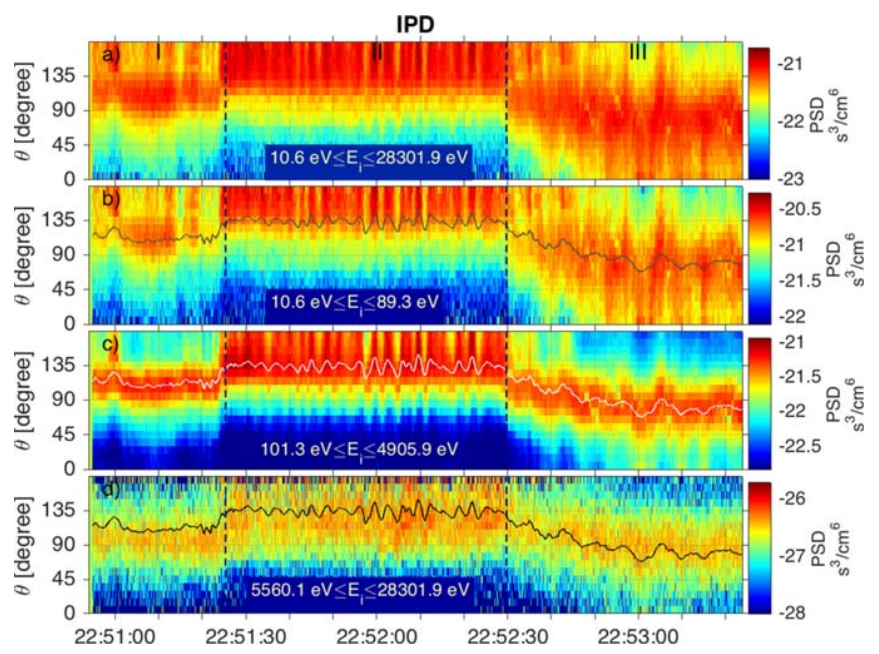

Figure 4. (a) IPD in the whole FPI ion energy range of [10.6 28301.9] eV. IPD in different energy range: (b) [10.6 89.3] eV, (c) [101.3 4905.9] eV, and (d) [5560.1 28301.9] eV. The solid lines overlaid in panels (b)-(d) represent the angle between the magnetic field and the ion bulk velocity.

pitch angle arises. The ion pitch angle periodically changes from $\sim 135^{\circ}-180^{\circ}$ to $\sim 90^{\circ}-135^{\circ}$, and this modulation period is agreement with the EMIC period. Because IPDs are computed in the spacecraft frame, most of changes in distributions result from changes in the angle between the magnetic field and the bulk ion flow. Therefore, the large-amplitude EMIC wave can modulate the IPDs (Zhao et al. 2018). In interval III, the ion pitch angle is mostly limited in the range of $\sim 45^{\circ}-135^{\circ}$. Also, there is a weak modulation for ions with the energy $101.3 \mathrm{eV} \leqslant E_{i} \leqslant 4905.9 \mathrm{eV}$ (Figure 4(c)), and the modulation period is nearly the same as the period of transverse magnetic field fluctuations (as shown in Figures 2(a)-(c)).

\subsection{Electron Dynamics}

Figure 5 shows the EPD. For low-energy electrons with $11.0 \mathrm{eV} \leqslant E_{e} \leqslant 33.5 \mathrm{eV}$, there are two kinds of motion behaviors. A part of electrons follow two dashed lines that represent $\theta=\cos ^{-1}\left(\left|B_{z}\right| /|B|\right)$ and $180-\theta$. These electrons propagate along and antiparallel to $B_{z}$ in GSE coordinates; however, the source for these low-energy electron enhancements is unclear. In intervals I and III, a part of electrons are limited in the angle range of $\theta_{c}<\theta<180^{\circ}-\theta_{c}$, where the critical pitch angle is defined as $\theta_{c}=\sin ^{-1}\left(\sqrt{|B| / B_{\max }}\right)$. These electrons are trapped by the magnetic structure relating to mirror mode waves.

For electrons with $38.0 \mathrm{eV} \leqslant E_{e} \leqslant 43.0 \mathrm{eV}$, a weak signature for electrons propagating along/counter to $B_{z}$ and for electrons trapping in $\theta_{c}<\theta<180^{\circ}-\theta_{c}$ exists. However, a major part of electrons are propagating parallel $\left(\theta<\theta_{c}\right)$ and antiparallel $\left(\theta>180^{\circ}-\theta_{c}\right)$ to the background magnetic field.

For $48.7 \mathrm{eV} \leqslant E_{e} \leqslant 70.7 \mathrm{eV}$, these electrons can be classified into two classes: trapped electrons $\left(\theta_{c}<\theta<180^{\circ}-\theta_{c}\right)$ and untrapped electrons $\left(\theta<\theta_{c}\right.$ and $\left.\theta>180^{\circ}-\theta_{c}\right)$. It is interesting to see that the heavily trapped electrons are cooling. This cooling is explained by the Fermi and Beatron deceleration in the development of the nonlinear mirror mode (Kivelson \& Southwood 1996; Breuillard et al. 2018).

For $80.1 \mathrm{eV} \leqslant E_{e} \leqslant 116.3 \mathrm{eV}$, in intervals I and II, the electron temperature seems nearly isotropic; however, a weak cooling arises for electrons with $\theta \sim 90^{\circ}$. In interval III,

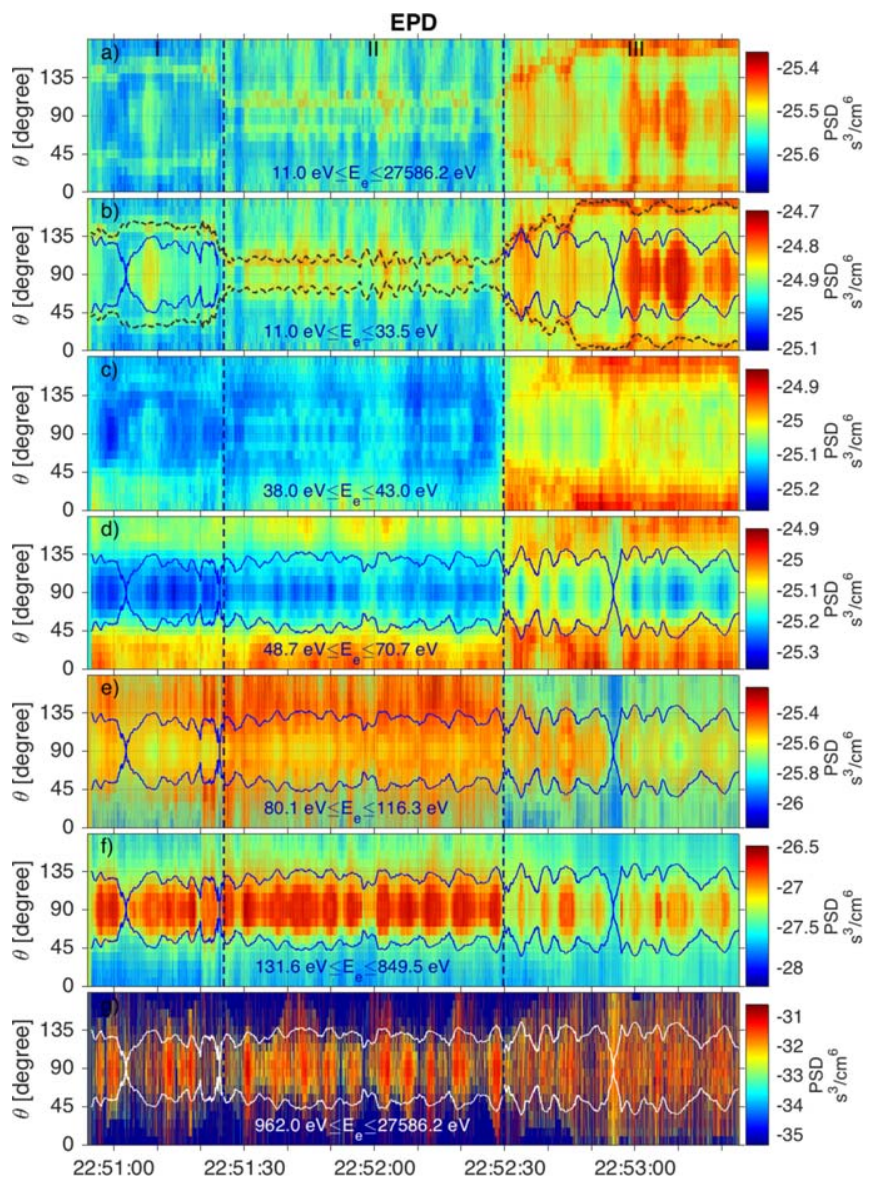

Figure 5. (a) EPD in the whole FPI electron energy range of [11.0 27586.2] eV. EPD in different energy range: (b) $\left[\begin{array}{lll}11.0 & 33.5\end{array}\right] \mathrm{eV}$, (c) $[38.043 .0] \mathrm{eV}$, (d) $[48.770 .7] \mathrm{eV}$, (e) [80.1 116.3] eV, (f) [131.6 849.5] eV, and (g) [962.0 $27586.2] \mathrm{eV}$. The dashed lines overlaid in panel (b) denote two angles defined as $\theta=\cos ^{-1}\left(\left|B_{z}\right| /|B|\right)$ and $180^{\circ}-\theta$. The solid lines represent two critical angles $\theta_{c}=\sin ^{-1}\left(\sqrt{|B| /|B|_{\max }}\right)$ and $180^{\circ}-\theta_{c}$.

besides the cooling for deeply trapped electrons, we find that shallowly trapped electrons are heated. The electron cooling and heating progresses can result from by Fermi acceleration/ deceleration and Beatron deceleration in the nonlinear evolution of the mirror mode (Kivelson \& Southwood 1996; Breuillard et al. 2018).

For electrons with $131.6 \mathrm{eV} \leqslant E_{e} \leqslant 849.5 \mathrm{eV}$ and even more energetic electrons $962.0 \mathrm{eV} \leqslant E_{e} \leqslant 27586.2 \mathrm{eV}$, most of the electrons are trapped. We do not find the cooling process for deeply trapped electrons as shown in Figures 5(d) and (e). These high-energy electrons have an anisotropic temperature with $T_{\perp}>T_{\|}$, which is the source of the observed whistler waves (Breuillard et al. 2018).

\section{Instability Analyses}

The observed waves in the magnetosheath normally stay at saturation, meaning that both wave excitation and wave damping happen. In order to investigate the source for these observed waves, here we give an instability analysis in the time interval 22:51:41.83 UT-22:51:43.14 UT, when whistler waves are strong and have the amplitude $\delta B \sim 1 \mathrm{nT}$. The averaged ion and electron phase space densities are presented in Figure 6. Figure 6 also gives the fitting ion and electron phase space densities. Through fitting these phase space 
(a) ION PHASE SPACE DENSITY

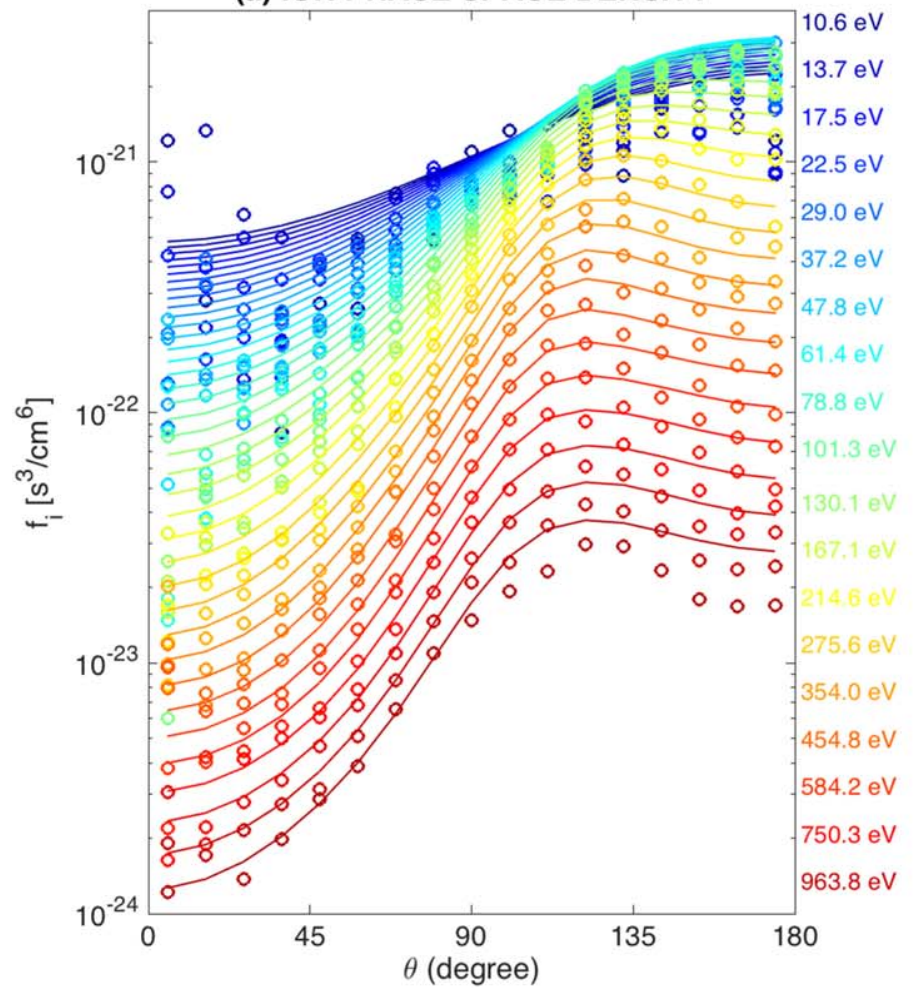

(b) ELECTRON PHASE SPACE DENSITY

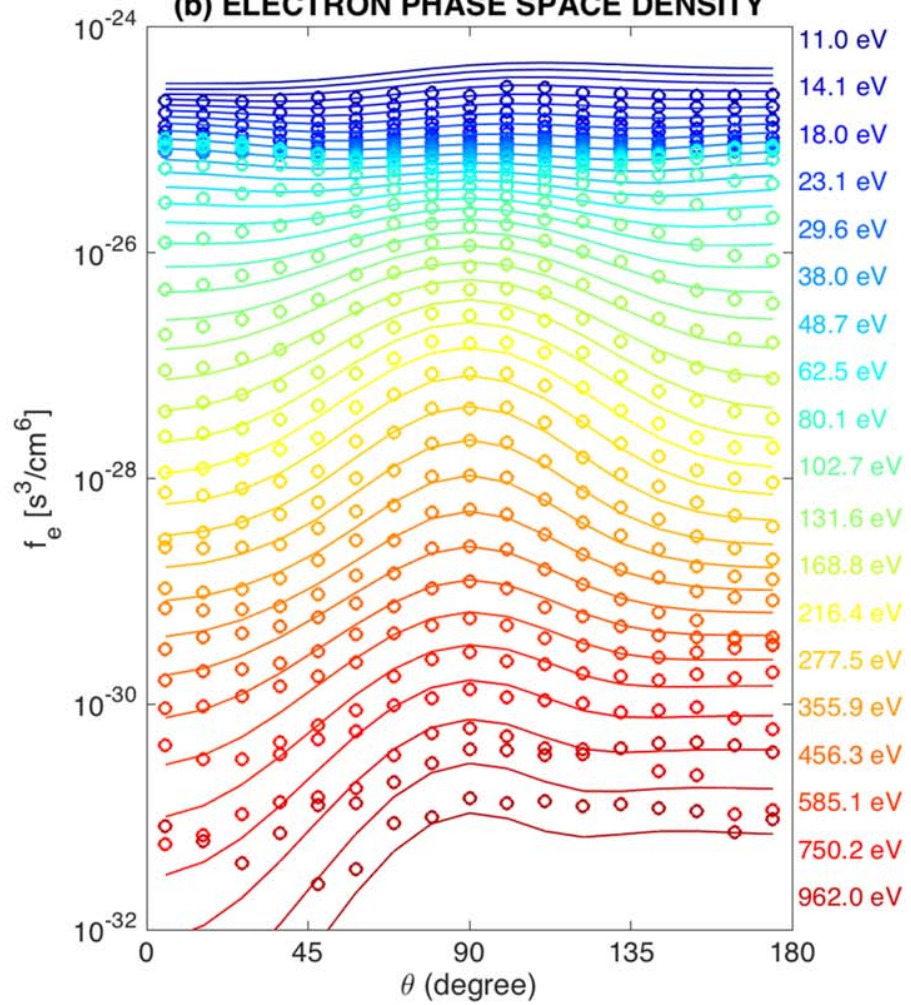

Figure 6. Ion and electron phase space densities. Circles represent the observed values in the time interval 22:51:41.83 UT-22:51:43.14 UT. The solid lines denote the fitting values.

densities, the plasma parameters are given in Tables 1 and 2 . Here, we assume the particle velocity distribution function following a drifting bi-Maxwellian distribution, $f\left(v_{\|}, v_{\perp}\right)=$ $n_{0}\left(\sqrt{\pi} V_{\mathrm{th} \|}\right)^{-3} T_{\|} T_{\perp}^{-1} \exp \left[-\left(v_{\|}-V_{d}\right)^{2} / V_{\mathrm{th} \|}^{2}\right] \exp \left(-v_{\perp}^{2} / V_{\mathrm{th} \perp}^{2}\right)$, where $V_{\mathrm{th} \|}=\sqrt{2 T_{\|} / m}$ is the parallel thermal velocity, $V_{\mathrm{th} \perp}=\sqrt{2 T_{\perp} / m}$ is the perpendicular thermal velocity, $V_{d}$ is the drifting velocity, $m$ is the particle mass, and $T_{\|}$and $T_{\perp}$ are the parallel and perpendicular temperatures, respectively. Furthermore, charge neutrality and zero current conditions are satisfied in fitting plasma parameters.

Based on the plasma kinetic model (Xie \& Xiao 2016) and the fitting plasma parameters, Figure 7 presents all linear instabilities, including the EMIC instability, mirror instability, and electromagnetic electron cyclotron instability. To illustrate the effects of streaming particles, theoretic predictions of linear instabilities in the motionless plasmas (neglecting the velocity drifting for all fitting ion and electron components) are represented by dashed lines in Figure 7. The wave direction is indicated by the sign of the wave frequency, i.e., $f>0$ and $f<0$, corresponding to the wave propagating along and against the magnetic field, respectively.

When the plasma is motionless, the EMIC instability produces parallel and antiparallel EMIC waves $\left(0^{\circ}\right.$ and $\left.180^{\circ}\right)$ with the same growth rate. The strongest excitation occurs at $f \simeq 0.56 f_{\mathrm{cp}} \simeq 0.32 \mathrm{~Hz}$, where the growth rate reaches the maximum of $\gamma_{\max } \simeq 0.071 f_{\mathrm{cp}} \simeq 0.040 \mathrm{~s}^{-1}$. Using our fitting plasma parameters, as $\lambda_{p} k>0.90$, the parallel-propagating wave becomes antiparallel propagating, where $\lambda_{p}$ is the proton inertial length defined by $\left(m_{p} / \mu_{0} n_{p C 1} e^{2}\right)^{1 / 2}$. Moreover, the effects of streaming ions would induce the antiparallel wave to grow stronger than the parallel wave. The strongest antiparallel
Table 1

Ion Fitting Parameters

\begin{tabular}{lcccc}
\hline \hline Component & $\begin{array}{c}\text { Density } \\
n_{i}\left(\mathrm{~cm}^{-3}\right)\end{array}$ & $\begin{array}{c}\text { Parallel } \\
\text { Temperature } \\
T_{\mathrm{i} \|}(\mathrm{eV})\end{array}$ & $\begin{array}{c}\text { Perpendicular } \\
\text { Temperature } \\
T_{\mathrm{i} \perp}(\mathrm{eV})\end{array}$ & $\begin{array}{c}\text { Drift Velocity } \\
V_{\mathrm{id}}\left(\mathrm{km} \mathrm{s}^{-1}\right)\end{array}$ \\
\hline $\mathrm{C} 1$ & 22.7 & 33 & 126 & -96.9 \\
$\mathrm{C} 2$ & 24.5 & 104 & 210 & -100.6 \\
$\mathrm{C} 3$ & 16.0 & 185 & 367 & -123.8 \\
$\mathrm{C} 4$ & 10.6 & 554 & 750 & -151.6 \\
\hline
\end{tabular}

Table 2

Electron Fitting Parameters

\begin{tabular}{lcccc}
\hline \hline Component & $\begin{array}{c}\text { Density } \\
n_{e}\left(\mathrm{~cm}^{-3}\right)\end{array}$ & $\begin{array}{c}\text { Parallel } \\
\text { Temperature } \\
T_{\mathrm{e} \|}(\mathrm{eV})\end{array}$ & $\begin{array}{c}\text { Perpendicular } \\
\text { Temperature } \\
T_{\mathrm{e} \perp}(\mathrm{eV})\end{array}$ & $\begin{array}{c}\text { Drift Velocity } \\
V_{\mathrm{ed}}\left(\mathrm{km} \mathrm{s}^{-1}\right)\end{array}$ \\
\hline $\mathrm{C} 1$ & 24.57 & 5 & 8 & -224.5 \\
$\mathrm{C} 2$ & 5 & 12 & 14 & 2054.4 \\
C3 & 6 & 11 & 15 & -2138.3 \\
C4 & 36 & 26 & 47 & 0 \\
C5 & 1.4 & 49 & 49 & 0 \\
C6 & 0.8 & 75 & 125 & 0 \\
C7 & 0.03 & 95 & 145 & -5930.6 \\
\hline
\end{tabular}

EMIC instability of $\gamma \simeq 0.11 f_{\mathrm{cp}} \simeq 0.062 \mathrm{~s}^{-1}$ occurs at $f \simeq$ $1.13 f_{\mathrm{cp}} \simeq 0.64 \mathrm{~Hz}$.

Also, both anisotropic ions and electrons are responsible for the excitation of the mirror instability (e.g., Remya et al. 2013). The zero-frequency mirror mode with the maximum growth rate of $\gamma_{\max } \simeq 0.086 f_{\mathrm{cp}} \simeq 0.049 \mathrm{~s}^{-1}$ is excited at $\theta \simeq 57^{\circ}$ in motionless plasmas. Streaming ions result in a shifted frequency for the mirror mode and slightly reduces its growth 
(a) EMIC INSTABILITY

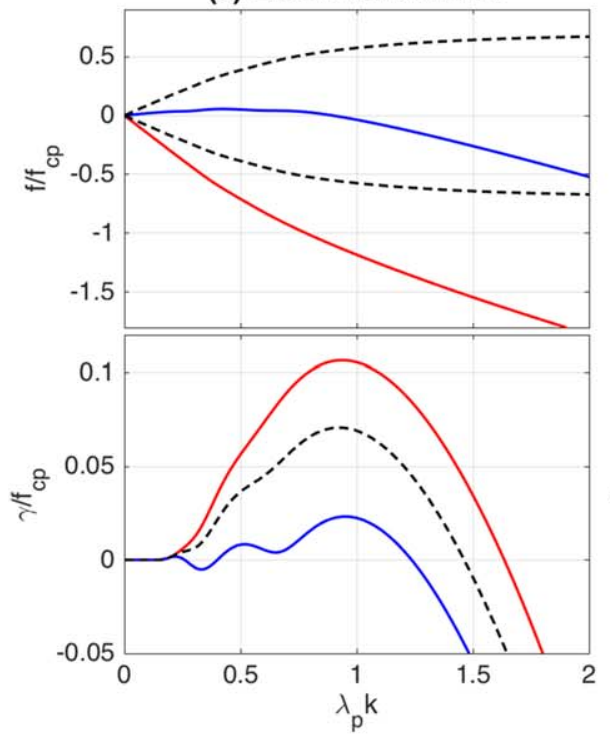

(b) MIRROR INSTABILITY

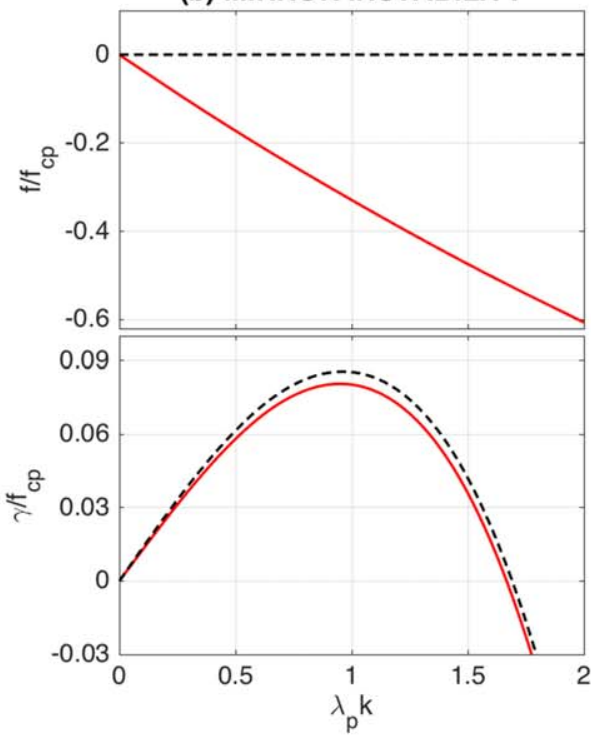

(c) EMEC INSTABILITY

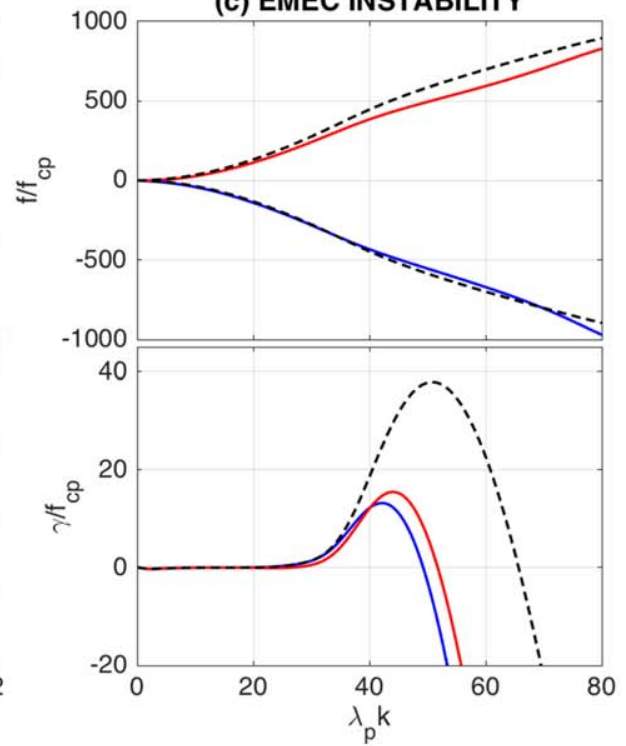

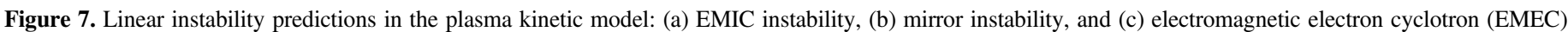
instability. The solid lines represent the results using the fitting plasma parameters. The dashed lines denote the results in motionless plasmas.

rate. Using our fitting parameters, the strongest mirror instability of $\gamma_{\max } \simeq 0.081 f_{\mathrm{cp}} \simeq 0.046 \mathrm{~s}^{-1}$ occurs at $\theta \simeq 58^{\circ}$, and the corresponding wave frequency is $f \simeq 0.31 f_{\mathrm{cp}} \simeq$ $0.17 \mathrm{~Hz}$

The whistler instability is mainly triggered by anisotropic electron components (i.e., the $n_{\mathrm{e} 4}$ component). In motionless plasmas, the electron cyclotron anisotropy instability produces parallel $\left(0^{\circ}\right)$ and antiparallel $\left(180^{\circ}\right)$ whistler waves with the same growth rate. The maximum growth rate of $\gamma_{\max } \simeq 38 f_{\mathrm{cp}} \simeq$ $21 \mathrm{~s}^{-1}$ arises at $f \simeq 595 f_{\mathrm{cp}} \simeq 336 \mathrm{~Hz}$. Streaming electrons result in an asymmetry of the growth rates of parallel and antiparallel whistler waves. Moreover, streaming electrons obviously reduce the wave frequency of the parallel wave, whereas the effect of streaming electrons weakly affects the wave frequency of the antiparallel wave. For parallel whistler waves, $\gamma_{\max } \simeq 15 f_{\mathrm{cp}} \simeq$ $8.5 \mathrm{~s}^{-1}$, which is slightly larger than the maximum growth rate of antiparallel whistler waves, $\gamma_{\max } \simeq 13 f_{\mathrm{cp}} \simeq 7.3 \mathrm{~s}^{-1}$. The wave frequency is $f \simeq 432 f_{\mathrm{cp}} \simeq 244 \mathrm{~Hz}$ for the most unstable parallel whistler wave and is $f \simeq 459 f_{\text {cp }} \simeq 259 \mathrm{~Hz}$ for the most unstable antiparallel wave. These theoretic results are qualitatively consistent with the observed features of whistler waves-for example, the parallel waves have the wave frequency lower than the antiparallel waves, and the former waves are stronger than the latter waves.

\section{Discussion and Summary}

This study provides a very interesting observational study on the dynamics of the plasma waves and particles in collisionless plasmas. In particular, our results have explored the important roles of streaming ions and electrons on EMIC and whistler waves in the Earth's magnetosheath.

As shown in Figure 2, EMIC waves only arise in interval II (not in intervals I and III), where the parallel streaming velocity of ions is larger than that in intervals I and III. From predictions of the linear instability theory in the plasma kinetic model (Figure 7), the growth rate of EMIC waves is larger than that of mirror mode waves as differential flows among ion components arise, whereas the EMIC growth rate is smaller than the mirror mode growth rate in motionless plasmas. Therefore, we propose that both effects of the ion temperature anisotropy and the ion differential flow considerably affect the existence the EMIC wave in the Earth's magnetosheath.

As shown in Figure 3, forward and backward whistler waves have different frequencies - that is, the frequencies of forward whistler waves are lower than the frequencies of backward waves. This could be a result of differential flows among electron components, as shown in Figure 7. Also, streaming electrons induce asymmetry of the growth rate of forward and backward whistler waves. Our fitting plasma parameters predict that the excited whistler waves have the normal angles of $\sim 0^{\circ}$ $30^{\circ}$ and $\sim 150^{\circ}-180^{\circ}$, and the strongest excitation occurs at parallel and antiparallel directions. This is different from the result proposed in $\mathrm{Li}$ et al. (2016), where highly oblique whistler waves $\left(\sim 70^{\circ}\right)$ are generated by electrons with streaming and anisotropic components. Li et al. (2016) use the fitting plasma parameters near the equator of the Earth's magnetosphere, which is quite distinct from our fitting plasma parameters. Therefore, the properties of whistler waves resulted from the streaming electron should be sensitive to the plasma parameters. On the other hand, using the equation from the quasi-linear theory in motionless plasmas (e.g., Kennel \& Petschek 1966; Lengyel-Frey et al. 1994), $E_{r}=$ $\frac{B_{0}^{2}}{2 \mu_{0} n_{e}} \frac{\Omega_{c e}}{\omega}\left(1-\frac{\omega}{\Omega_{c e}}\right)\left(1+\frac{\omega}{\Omega_{c e}}\right)^{2}$ (in the unit of kelvin), we can calculate the energy of electrons that suffer the normal cyclotron resonance with observed whistler waves-that is $E_{r} \sim 1.6-7.9 \mathrm{keV}$ for whistler waves with $\omega / \Omega_{c e} \sim 0.1-0.5$ (Figure 3).

Also, our observations have explored particle pitch angle distributions in the presence of EMIC, mirror and whistler waves. In time interval II, large-amplitude EMIC waves result in a modulation in the IPD. In interval III, transverse electromagnetic fluctuations $\left(\left|\boldsymbol{B}_{\perp}\right| / B_{0} \simeq\left|V_{i \perp}\right| / V_{\mathrm{A}}\right)$ play a role in modulating the pitch angle of ions with the energy of $101.3 \mathrm{eV}<E_{i}<4905.9 \mathrm{eV}$. These modulations mainly result from magnetic structures associated with EMIC (or Alfvénic) waves. For electrons, they are predominately affected by mirror 
mode waves. Because of the mirror magnetic structure, electrons are preferentially trapped in magnetic troughs. As explored in previous works (e.g., Chisham et al. 1998; Ahmadi et al. 2018; Breuillard et al. 2018; Yao et al. 2018), the shallowly (deeply) trapped electrons can have heating (cooling) due to Fermi acceleration (Fermi and Beatron cooling). However, in time intervals I and III, low-energy electrons are heavily trapped in magnetic troughs, which have not been found in previous works (e.g., Ahmadi et al. 2018; Breuillard et al. 2018). Also, this trapping for low-energy electrons does not seem to be consistent with the physical picture of the nonlinear development of the mirror mode, which predicts cooling for low-energy trapped electrons. Therefore, our results would motivate further theoretical investigations on the electron dynamics in the mirror mode structure.

This work was supported by the NNSFC 41531071, 11673069, and 11761131007; and by NSF of Jiangsu Province under Grant BK20161617. T.Y.W. is supported by the Marie Skodowska Curie Grant 665593 from the European Union's Horizon 2020 Research and Innovation Programme. D.B.G. is supported by Swedish National Space Board, grant 128/17. M. W.D. is supported by NNSFC 41874193 and 41574155 , NERC Grant NE/H004076/1, and by STFC in-house research grant. J.S.H. is supported by NNSFC 41574168 and 41874200 . Portions of this work were performed at the Jet Propulsion Laboratory, California Institute of Technology under contract with NASA. We thank the MMS team for data access and support, and the data are available at the LASP-CU Boulder website (https://lasp.colorado.edu/mms/sdc/public/).

\section{ORCID iDs}

Jinsong Zhao (iD https://orcid.org/0000-0002-3859-6394

\section{References}

Ahmadi, N., Wilder, F. D., Ergun, R. E., et al. 2018, JGRA, 123, 6383 Baumjohann, W., Treumann, R. A., Georgescu, E., et al. 1999, AnGeo, 17,1528

Breuillard, H., Le Contel, O., Chust, T., et al. 2018, JGRA, 123, 93

Brice, N. 1964, JGR, 69, 4515
Chisham, G., Burgess, D., Schwartz, S. J., \& Dunlop, M. W. 1998, JGR, 103, 26765

Dimmock, A. P., Osmane, A., Pulkkinen, T. I., \& Nykyri, K. 2015, JGRA, 120, 5489

Ergun, R. E., Tucker, S., Westfall, J., et al. 2016, SSRv, 199, 167

Gary, S. P. 1992, JGR, 97, 8519

Gary, S. P., Fuselier, S. A., \& Anderson, B. J. 1993, JGR, 98, 1481

Giagkiozis, S., Wilson, L. B., Burch, J. L., et al. 2018, JGRA, 123, 5435

Hasegawa, A. 1969, PhFl, 12, 2642

Hoilijoki, S., Palmroth, M., Walsh, B. M., et al. 2016, JGRA, 121, 4191

Huang, S. Y., Du, J. W., Sahraoui, F., et al. 2017a, JGRA, 122, 8577

Huang, S. Y., He, L. H., Yuan, Z. G., et al. 2019, ApJ, 875, 113

Huang, S. Y., Sahraoui, F., Yuan, Z. G., et al. 2017b, ApJL, 836, L27

Huang, S. Y., Sahraoui, F., Yuan, Z. G., et al. 2018, ApJ, 861, 29

Karimabadi, H., Roytershteyn, V., Vu, H. X., et al. 2014, PhP1, 21, 062308

Kennel, C. F., \& Petschek, H. E. 1966, JGR, 71, 1

Kivelson, M. G., \& Southwood, D. J. 1996, JGR, 101, 17365

Le Contel, O., Leroy, P., Roux, A., et al. 2016, SSRv, 199, 257

Lee, K. H. 2017, JGRA, 122, 7307

Lee, L. C., Wu, C. S., \& Price, C. P. 1987, JGR, 92, 2343

Lengyel-Frey, D., Farrell, W. M., Stone, R. G., Balogh, A., \& Forsyth, R. 1994, JGR, 99, 13325

Li, W., Mourenas, D., Artemyev, A. V., et al. 2016, GeoRL, 43, 8867

Lindqvist, P.-A., Olsson, G., Torbert, R. B., et al. 2016, SSRv, 199, 137

Maksimovic, M., Harvey, C. C., Santolik, O., et al. 2001, AnGeo, 19, 1429

Masood, W., Schwartz, S. J., Maksimovic, M., \& Fazakerley, A. N. 2006, AnGeo, 24, 1725

Midgley, J. E., \& Davis, L., Jr. 1963, JGR, 68, 5111

Pollock, C., Moore, T., Jacques, A., et al. 2016, SSRv, 199, 331

Price, C. P., Swift, D. W., \& Lee, L.-C. 1986, JGR, 91, 101

Remya, B., Reddy, R. V., Tsurutani, B. T., Lakhina, G. S., \& Echer, E. 2013 JGR, 118, 785

Russell, C. T., Anderson, B. J., Baumjohann, W., et al. 2016, SSRv, 199, 189

Santolík, O., Parrot, M., \& Lefeuvre, F. 2003, RaSc, 38, 1010

Smith, E. J., Holzer, R. E., \& Russell, C. T. 1969, JGR, 74, 3027

Smith, E. J., \& Tsurutani, B. T. 1976, JGR, 81, 2261

Thorne, R. M., \& Tsurutani, B. T. 1981, Natur, 293, 384

Treumann, R. A., \& Baumjohann, W. 1997, Advanced Space Plasma Physics (London: Imperial College Press)

Tsurutani, B. T., \& Lakhina, G. S. 1997, RvGeo, 35, 491

Tsurutani, B. T., \& Rodriguez, P. 1981, JGR, 86, 4317

Tsurutani, B. T., Smith, E. J., Anderson, R. R., et al. 1982, JGR, 87, 6060

Xie, H., \& Xiao, Y. 2016, PIST, 18, 97

Yao, S. T., Shi, Q. Q., Liu, J., et al. 2018, JGRA, 123, 5561

Zhang, Y., Matsumoto, H., \& Kojima, H. 1998, JGR, 103, 4615

Zhao, J. 2015, PhPl, 22, 042115

Zhao, J. S., Wang, T. Y., Dunlop, M. W., et al. 2018, ApJ, 867, 58

Zhao, J. S., Wang, T. Y., Dunlop, M. W., et al. 2019, GeoRL, 46, 4545

Zwan, B. J., \& Wolf, R. A. 1976, JGR, 81, 1636 